\title{
Profiling of Mycoplasma gallisepticum Ribosomes
}

\author{
G. Y. Fisunov, D. V. Evsyutina, A. A. Arzamasov, I. O. Butenko, V. M. Govorun \\ Scientific research institute of physical-chemical medicine, Malaya Pirogovskaya, 1a, 119435, \\ Moscow, Russia \\ E-mail: herr.romanoff@gmail.com \\ Received 16.06.2015 \\ Copyright $\odot 2015$ Park-media, Ltd. This is an open access article distributed under the Creative Commons Attribution License,which permits \\ unrestricted use, distribution, and reproduction in any medium, provided the original work is properly cited.
}

\begin{abstract}
The development of high-throughput technologies is increasingly resulting in identification of numerous cases of low correlation between mRNA and the protein level in cells. These controversial observations were made on various bacteria, such as E. coli, Desulfovibrio vulgaris, and Lactococcus lactis. Thus, it is important to develop technologies, including high-throughput techniques, aimed at studying gene expression regulation at the level of translation. In the current study, we performed proteomic profiling of $M$. gallisepticum ribosomes and identified high abundant noncanonical proteins. We found that binding of mRNAs to ribosomes is mainly determined by two parameters: (1) abundance of mRNA itself and (2) complimentary interactions between the 3 ' end of $16 \mathrm{~S}$ rRNA and the ribosome binding site in the 5'-untranslated region of mRNA.
\end{abstract}

KEYWORDS mycoplasma, ribosome, ribosome profiling.

\section{INTRODUCTION}

System research using the so-called "omics technologies" increasingly reveal unexpected phenomena and new regulated events that simply cannot be determined using either an omics technology alone or traditional methods of analysis. However, the joint analysis of data obtained from the quantitative measurement of RNA or protein and peptide levels inevitably creates a significant number of artifacts due to mistakes in each of the methods used. This fact requires careful cross analysis and additional confirmation of the obtained data using alternative methods or orthogonal processing. For this reason, prokaryotes are used as subjects for testing a common methodology for the analysis of the joint behavior of macromolecules in living systems and their reciprocal impact.

The European Molecular Biology Laboratory has established a project dedicated to studying the causative agent of human respiratory diseases, a representative of the class Mollicutes - Mycoplasma pneumoniae [1, 2]. Then, our group selected another representative of this class (M. gallisepticum) to carry out a similar study. Soon afterwards, American researchers developed a computer model of the metabolism and adaptive responses of the smallest self-replicating bacteria $-M$. genitalium [3]. Despite these successes, a large number of issues remains unexplored, requiring additional methods for accessing dynamic processes during expression of the minimum set of genetic information encoded in the genome of Mycoplasma.
Mollicutes, which include M. gallisepticum, are characterized by a significant genome reduction. The average size of the genome of Mycoplasma typically ranges from 800 thousand to 1 million bp ( 1 million in M. gallisepticum) [4]. Due to genome reduction, Mycoplasma lost the well-known mechanisms of regulation of gene expression [5].

As we have showed earlier, M. gallisepticum responds to stress at the transcriptional level [6]. At the same time, these changes are generally slightly reflected at the level of translation [6]. This phenomenon may be caused by two reasons: (1) the rate of translation in M. gallisepticum is not enough to reveal changes in the protein level during the experiment (30 $\mathrm{min}$ ), (2) mRNA binds selectively to ribosomes during the stress response. The mechanism of selective attaching of mRNA to ribosomes can be realized through interaction with antisense RNA that blocks the ribosome binding site [7]. Moreover, even within the same cell, ribosomes may differ from each other both in the nucleotide sequence of rRNA [8] and in protein composition. For example, ribosomes in Escherichia coli, which do not include $\mathrm{S} 1$ protein, translate mainly leaderless transcripts [9]. Ribosomes may bind regulatory proteins modulating translation in particular transcripts [10]. Ribosomes may bind noncanonical proteins whose primary function is not related to translation. For example, glycogen synthase in Sascharomyces cerevisiae can affect translation of various RNAs [11]. 
The development of high-throughput technologies has led to the accumulation of a large database on transcription and translation in the entire cell. According to the classical view, protein level is generally determined by the level of the corresponding mRNA; however, in some cases, it is not. High-throughput technologies have increased the number of cases when the protein level does not correlate with the level of mRNA. Such data were obtained for a variety of bacteria, including E. coli [12], Desulfovibrio vulgaris [13], and Lactococcus lactis [14]. The Pearson correlation coefficient between the level of mRNA and protein level, according to the published data, can vary from 0.53 to 0.19 , depending on the type and state of the bacteria. Significant progress in studying the regulation of gene expression at the translational level was achieved with a technology of ribosome profiling [15] which enables observing translation almost in real time. Thus, the stage of binding $\mathrm{mRNA}$ to the ribosome and the process of translation is an extremely significant part in the regulation of gene expression in bacteria. Since Mollicutes in general and M. gallisepticum in particular are characterized by the lack of transcriptional regulatory mechanisms, regulation of gene expression at the translational level can be perhaps the most significant part in determining protein abundance in the cell.

In the present study, a high-throughput proteomic profiling of M. gallisepticum ribosomes to determine the composition of ribosomes and transcriptional profiling of ribosome-bound mRNA using real-time PCR were performed.

\section{EXPERIMENTAL SECTION}

\section{M. gallisepticum $\mathbf{S 6}$ culturing}

M. gallisepticum $\mathbf{S} 6$ was cultured in a liquid medium (20 g/l Tryptose, $5 \mathrm{~g} / 1 \mathrm{NaCl}, 1.3 \mathrm{~g} / 1 \mathrm{KCl}, 3 \mathrm{~g} / 1$ Tris, $5 \%$ yeast dialysate, $6 \%$ horse serum, and $1 \%$ glucose; $\mathrm{pH}$ 7.4) to mid-logarithm growth phase as described in [16].

\section{Ribosome purification}

Chloramphenicol was added to $12 \mathrm{ml}$ of M. gallisepticum cell culture to a final concentration of the solution of $100 \mu \mathrm{g} / \mathrm{ml}$, which was thoroughly mixed and incubated for $5 \mathrm{~min}$ on ice. Then, the cells were pelleted by centrifugation at $4,500 \mathrm{~g}$ for $20 \mathrm{~min}\left(4^{\circ} \mathrm{C}\right)$. The supernatant was removed, and the cell pellet, which was obtained from $50 \mathrm{ml}$ of culture, was resuspended in 500 $\mu \mathrm{l}$ of lysis buffer containing $20 \mathrm{mM}$ HEPES, $100 \mathrm{mM}$ $\mathrm{NaCl}, 6 \mathrm{mM} \mathrm{MgCl}{ }_{2}, 2 \mathrm{mM}$ spermidine, $100 \mu \mathrm{g} / \mathrm{ml}$ chloramphenicol, $5 \mu \mathrm{l}$ protease inhibitor (GE Healthcare), and 200 units of RNase inhibitor (Thermo Scientific) ( $\mathrm{pH}$ 7.5). Following the resuspension, $15 \mu \mathrm{l}$ of NP-40 was added to the buffer and the composition was mixed
Table 1. Spearman correlation between biological replicates of ribosome-bound mRNA sample. Levels of mRNAs were measured by real-time PCR

\begin{tabular}{|c|c|c|c|}
\hline & Rep1 & Rep2 & Rep3 \\
\hline Rep1 & 1 & 0.87 & 0.92 \\
\hline Rep2 & 0.87 & 1 & 0.90 \\
\hline Rep3 & 0.92 & 0.90 & 1 \\
\hline
\end{tabular}

thoroughly. Then, the cell lysate was frozen for at least 1 hour at $-75^{\circ} \mathrm{C}$. The cell lysate was purified by centrifugation at $20,000 \mathrm{~g}$ for $20 \mathrm{~min}\left(4^{\circ} \mathrm{C}\right)$. The supernatant was collected and fractionated by centrifugation on a sucrose step gradient.

Sucrose step gradient was created in a $5 \mathrm{ml}$ polycarbonate tube by layering sucrose solutions of different densities using a pipette. The volume of each layer was $750 \mu \mathrm{l}$, and the difference in density was $10 \%$. In this study, we used 10-50\% sucrose gradients (a total of 5 layers). Sucrose solution was prepared using the same buffer as for cell lysis (without adding NP-40, chloramphenicol, and inhibitors of proteases and RNases). The mixture was centrifugated at $50,000 \mathrm{rpm}(200,620 \mathrm{~g}$ on average) for $1 \mathrm{~h}$ at $4^{\circ} \mathrm{C}$, using the Optima centrifuge (Beckman Coulter) and the MLS 50 swinging bucket rotor (Beckman Coulter). $200 \mu \mathrm{l}$ aliquots of fractions were collected using a pipette.

\section{RNA isolation from fractions}

Each fraction was added to $400 \mu \mathrm{l}$ of Trizol LS reagent (Life Technologies). The content was thoroughly mixed, and $200 \mu \mathrm{l}$ of chloroform was added. The composition was then mixed again and centrifuged for 10 minutes at $16,000 \mathrm{~g}\left(4^{\circ} \mathrm{C}\right)$. The supernatant was collected and resuspended in an equal volume of isopropanol. The sample was incubated for at least $1 \mathrm{~h}$ at $-20^{\circ} \mathrm{C}$. RNA was pelleted by centrifugation at $16,000 \mathrm{~g}$ for $20 \mathrm{~min}$ $\left(4^{\circ} \mathrm{C}\right)$. The pellet was washed with $80 \%(\mathrm{v} / \mathrm{v})$ ethanol. The RNA sample was then dissolved in $10 \mu$ l of water (Panreac). RNA abundance in the fractions was measured by quantitative real-time PCR. The experiment was conducted in 3 biological replicates.

\section{Protein extraction from fractions}

and trypsin digestion

For protein precipitation, each fraction was diluted 10 -fold with deionized water and trichloroacetic acid (Sigma-Aldrich) was added to a final concentration of $10 \%(\mathrm{v} / \mathrm{v})$. The mixture was left at $4^{\circ} \mathrm{C}$ overnight and 

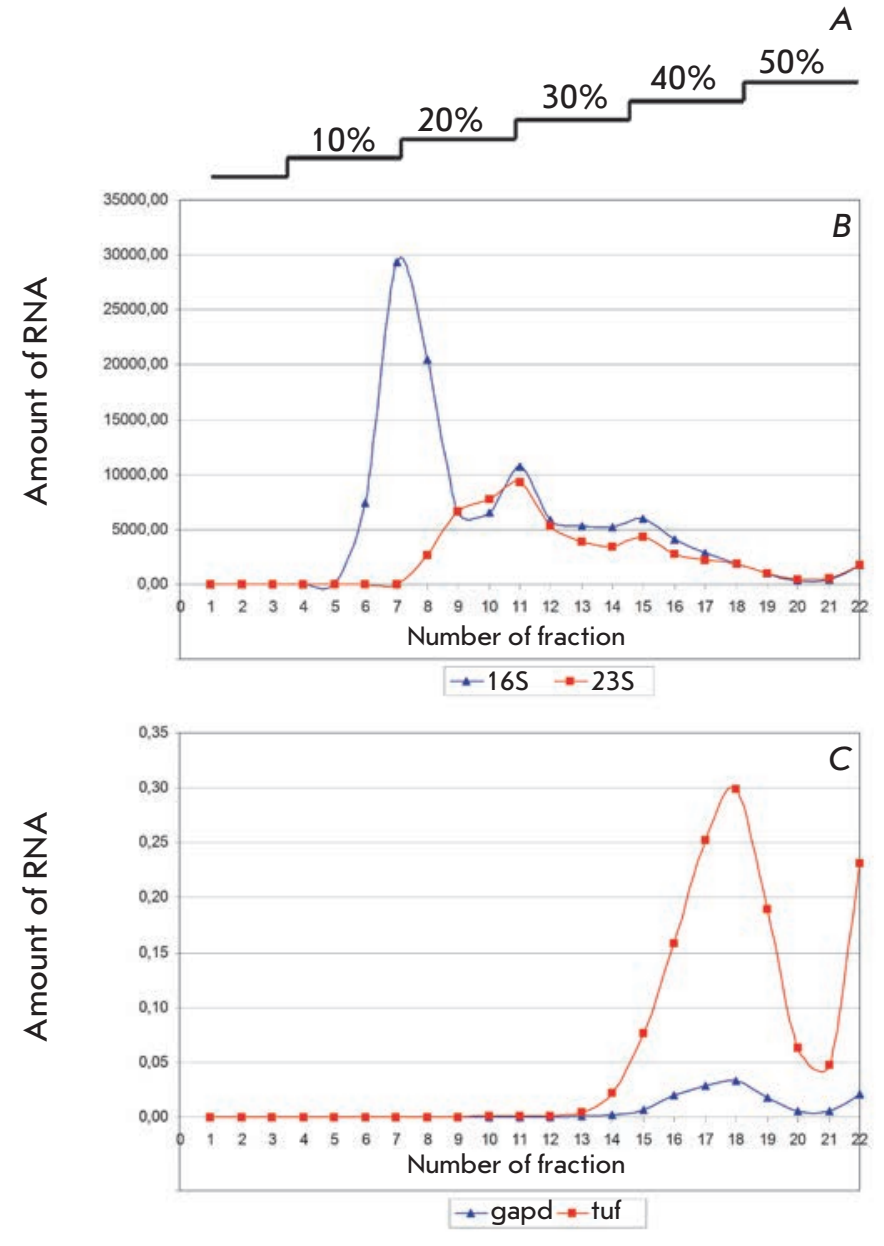

Fig. 1. Fractionation of cytoplasm of M. gallisepticum in a sucrose gradient. Abundance of 16S rRNA, 23S rRNA, gapd mRNA, and tuf mRNA was measured. All results (for rRNA and mRNA) were normalized to the 16S mRNA level in fraction 1. A - step gradient of sucrose (regarding obtained fractions); $\mathrm{B}$ - abundance of rRNA; $\mathrm{C}-$ abundance of mRNA

then centrifugated for $15 \mathrm{~min}$ at $16,000 \mathrm{~g}$. The pellet was washed twice with $1 \mathrm{ml}$ of cold acetone (Pancreac) to remove residual trifluoroacetic acid.

Protein pellets were redissolved in 25-35 $\mu$ l of 50 $\mathrm{mM}$ ammonium bicarbonate solution (Pancreac) containing $0.5 \%$ RapiGest SF (Waters) and $1 \mu$ of Nuclease Mix (GE Healthcare). Then, the mixture was left for $30 \mathrm{~min}$ at $4^{\circ} \mathrm{C}$, incubated for $5 \mathrm{~min}$ at $100^{\circ} \mathrm{C}$, and centrifuged for $10 \mathrm{~min}$ at $16,000 \mathrm{~g}$. The supernatant was collected, and the protein content was determined in each sample using bicinchoninic acid (Bicinchoninic Acid Protein Assay Kit, Sigma-Aldrich). In order to reduce disulfide bonds, dithiothreitol (Bio-Rad) was added to the protein solution to a final concentration of $10 \mathrm{mIM}$ (the reaction was conducted on a shaker $(600$ rpm) for 30 minutes at $60^{\circ} \mathrm{C}$ ). The subsequent alkylation of cysteine residues by iodoacetamide (final concentration of $30 \mathrm{mM}$; Bio-Rad) was performed for $30 \mathrm{~min}$ at room temperature in the dark. Then, trypsin (Trypsin Gold, Mass Spectrometry Grade, Promega) was added to protein samples; trypsin:protein ratio $(\mathrm{w} / \mathrm{w})$ was 1:50. Trypsin digestion was performed during 16 hours at $37^{\circ} \mathrm{C}$. The reaction was stopped by adding $10 \%$ trifluoroacetic acid (Sigma-Aldrich) (pH after trifluoroacetic acid addition should be 2.0 ). Then, the sample was incubated for $45 \mathrm{~min}$ at $37^{\circ} \mathrm{C}$ and centrifuged $(15$ min at $16,000 \mathrm{~g}$ ) to remove RapiGest $\mathrm{SF}$. The mixture of tryptic peptides was additionally purified by solid phase extraction using Discovery DSC-18 mini columns (Supelco) according to the manufacturer's recommendations. For further mass spectrometry analysis, the eluate was dried in the CentriVap vacuum concentrator (Labconco) and dissolved in $10 \mu \mathrm{l}$ of $3 \%$ acetonitrile solution containing $0.1 \%$ formic acid.

RNA isolation from cell culture

RNA was extracted from the cell culture according to [16]. Triple volume of Trizol LS reagent (Thermo Scientific) was added to aliquotes of cell culture. Phase separation was induced by adding chloroform (80 $\mu \mathrm{l}$ per $100 \mu \mathrm{l}$ of cell culture). Samples were centrifuged at $10,000 \mathrm{~g}$ for $15 \mathrm{~min}\left(4^{\circ} \mathrm{C}\right)$. Then, the RNA samples were reprecipitated with isopropanol $(1: 1 \mathrm{v} / \mathrm{v})$.

cDNA synthesis and qPCR

cDNA synthesis and real-time PCR were performed as described in [16]. RNA samples were treated with DNase I (Thermo Scientific). Then, cDNA was synthesized with reverse transcriptase ( $\mathrm{H}$ minus Reverse Transcriptase, Thermo Scientific) and random hexamers. RiboLock RNase inhibitor (Thermo Scientific) was used to enhance the stability of RNA.

Quantitative real-time PCR (qRT-PCR) was performed on the C1000 Touch thermal cycler (Bio-Rad) with the CFX96 optical module (Bio-Rad). For PCR, 10X PCR buffer (Lytech) (1.5-fold final dilution), 10X dNTP mixture (Lytech), Taq-polymerase (Lytech), SYBR Green I dye (Life Technologies), 5 pmol primers, and $2 \%$ formamide were used. Data normalization was carried out according to the average $\mathrm{mRNA}$ abundance in 21 housekeeping genes (eno, gaphd, tpiA, tuf, tsf, aco $A, a c o B, a c e F, l d h, a c k A, p g k, f b a, p g i, p f k A, g p m I$, $p y k F, t k t A 1, \operatorname{rpiB}$, eutD, prs $A$, and $l p d)$. The same as in [6] primers were used for qRT-PCR.

\section{Identification of Proteins}

Chromatography-mass spectrometry analysis of peptide extracts was performed using the Q-Exactive $\mathrm{HF}$ mass spectrometer (Thermo Fisher Scientific) coupled 
with the Ultimate 3000 RSLCnano LC system (Dionex) through the Nanospray Flex ion source (Thermo Fisher Scientific).

Peptides were separated by inversed-phase chromatography using Acclaim PepMap precolumn (C18 stationary phase, length of $2 \mathrm{~cm}, 75 \mathrm{~mm}$ i.d., particle size of $3 \mu \mathrm{m}$, and pore size of $100 \mathrm{~A}$; Dionex) and Zorbax column (Zorbax 300SB-C18 stationary phase, length of $15 \mathrm{~cm}, 75 \mathrm{~mm}$ i.d., particle size of $3.5 \mathrm{~mm}$, and pore size of $100 \mathrm{~A}$; Agilent Technologies). Each sample was applied to the precolumn in water for high-pressure liquid chromatography (HPLC) with $0.1 \%$ formic acid (v/v) for 5 minutes at a flow rate of $2 \mu \mathrm{l} / \mathrm{min}$. Then, the precolumn was placed in front of the column. Peptides were eluted with a mixture of solvent $A$ (water for HPLC with $0.1 \%$ formic acid (v/v)) and solvent B (79.9\% acetonitrile for HPLC (v/v), 20\% water for HPLC, and $0.1 \%$ formic acid (v/v)), increasing the density gradient of solvent B from 5 to $40 \%(\mathrm{v} / \mathrm{v})$ for 120 minutes at a flow rate of $300 \mathrm{nl} / \mathrm{min}$. Then, the system was washed for 10 minutes with a mixture of $99 \%$ solvent $B(v / v)$ and for 10 minutes with a mixture of $5 \%$ solvent $\mathrm{B}$.

The voltage was $2000 \mathrm{~V}$; the temperature of the capillary was $200^{\circ} \mathrm{C}$. The mass spectrometer operated in a data-dependent acquisition mode: in each cycle, a panoramic spectrum was obtained; the most intense 20 peaks in the panoramic spectrum were selected in turn for fragmentation and recording of product ion spectra and then excluded from the assessment for 10 seconds. A panoramic spectrum was recorded at a resolution of 70,000 in a mass-to-charge ratio value of 400 to 1200 $\mathrm{m} / \mathrm{z}$ with automatic gain control (AGC) setting of 106 and time limits for filling of $50 \mathrm{~ms}$. Product ion spectra were recorded at the resolution of 17,500 and AGC of 105 with time limits for filling of $100 \mathrm{~ms}$. Collision energy was $30 \mathrm{~V}$; the width of the ion isolation window was $2 \mathrm{~m} / \mathrm{z}$.

On the basis of mass chromatograms (.raw file format), a list of centroid spectra in Mascot Generic Format using the MSConvert utility of the ProteoWizard package (version 3.0.7.414, 64 bits) was compiled, which was then interpreted by the Mascot search engine (Matrix Science Inc.). Protein identification was carried out using the CP006916.2 protein sequence database of M. gallisepticum S6, which was supplemented by sequences for common protein contaminants. The following parameters of identification were used: tryptic peptides; maximum of one missed cleavage; precursor-ion charge was +2 or +3 ; the allowable error in the mass of parent ions was $10 \mathrm{ppm}$; the accuracy of the mass peak measurement of fragments was 0.5 Da; ESITRAP instrument; no permanent modification; cysteine carbamidomethylation and oxidation of methionine residues were variable modifications.

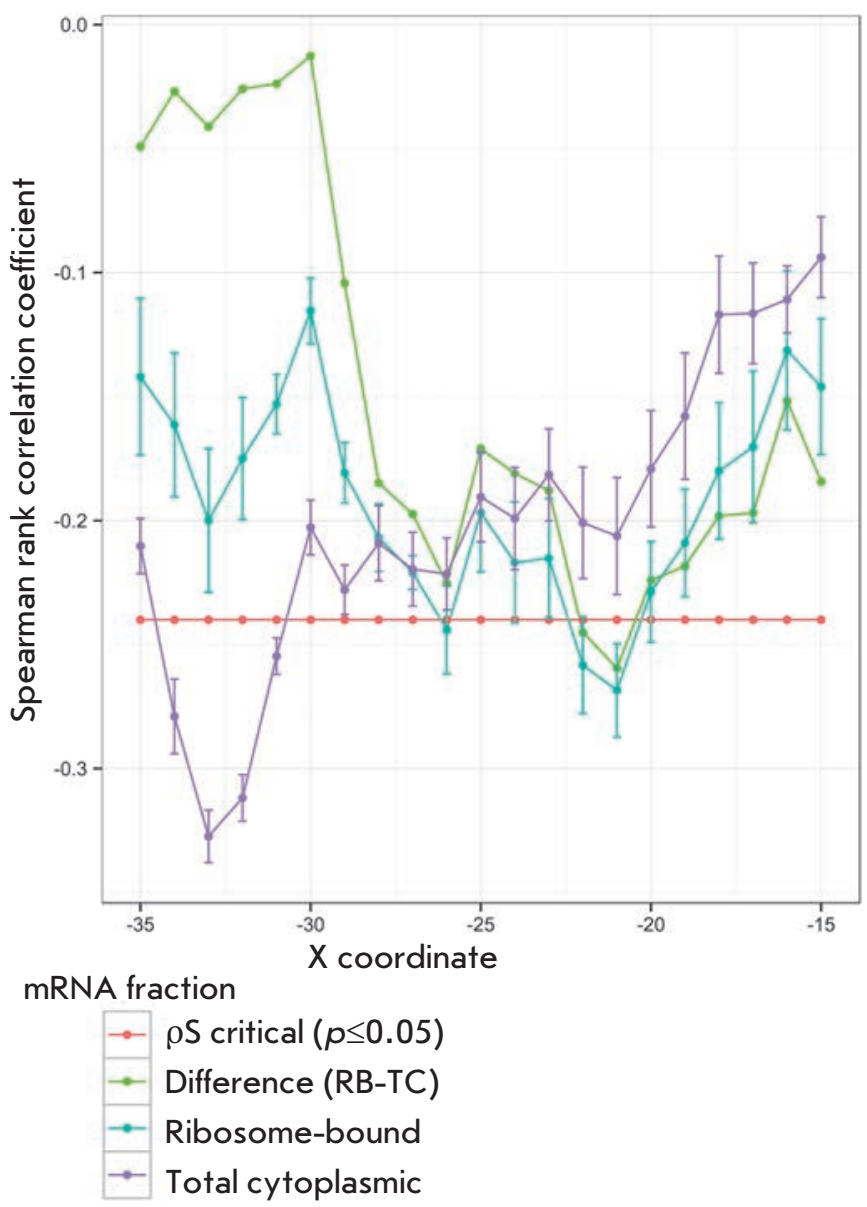

Fig. 2. Correlation between mRNA abundance in a given fraction and dG of the secondary structure near the start codon in a sliding window of $30 \mathrm{nt}(\mathrm{p}<0.05$ for $\rho>0.25)$

The list of statistically significant identities was defined as a list of proteins with 2 or more identified peptides with $\mathrm{p}<0.05$.

\section{RESULTS AND DISCUSSION}

Distribution of RNA in isolated fractions

After the cytoplasm of $M$. gallisepticum was fractionated, distribution of RNA in the fractions was measured by RT-qPCR (see Experimental section). The results are presented in Fig. 1. Small ribosomal subunits and large ones cause peaks in fractions 7 and 11, respectively. M. gallisepticum is characterized by anapproximately 4-fold abundance of $16 \mathrm{~S}$ rRNA compared to 23S rRNA [16], which is consistent with the observed picture. Fractions after 12-22 reveal an equimolar ratio of $16 \mathrm{~S}$ and $23 \mathrm{~S}$ rRNA. Relatively high levels of mRNA was detected only in polysomes (fractions 15 
and above). The highest amount of mRNA was detected in fractions 17 and 18, comprizing about 1 microgram. This makes the corresponding fractions most suitable for further analysis, especially when using high-throughput sequencing technologies.

Suitability of the technique for the quantitative analysis of the abundance of transcripts associated with ribosomes was tested for fraction 18. Transcriptional profiling of 67 genes in three biological replicates using RT-qPCR was conducted. The reproducibility of the data was evaluated using Spearman correlation (see table). In each pair of samples, the correlation coefficient ranged from 0.87 to 0.92 , indicating good reproducibility of the method. The correlation between the abundance of transcripts in the ribosome-bound mRNA fraction and total cytoplasmic mRNA fraction was 0.78 .

\section{Proteomic profiling of $M$. gallisepticum ribosomes}

In order to validate the methods of ribosome purification from M. gallisepticum, proteomic profiling of fractions 7 (30S subunit), 17, and 18 ( 70 S ribosomes associated with the mRNA) was conducted and a semiquantitative evaluation of protein abundance according to emPAI was made.

In fraction 7,18 out of 20 proteins of the small ribosome subunit and only 8 out of 33 proteins of the large subunit were detected, which agrees with the data on the distribution of rRNA in fractions. Thus, fraction 7 was mainly composed of small ribosome subunits. It should be noted that fraction 7 included a significant amount of additional cellular proteins.

Ribosomal proteins were mostly abundant, as expected, in fractions 17-18. A total of 47 out of 53 ribosomal proteins (19 out of 20 proteins of 30 S subunit and 28 out of 33 proteins of 50 S subunit) were detected. All proteins that were not identified were small in size (less than 100 amino acids); the latter probably impeded identification of these proteins. The abundance of ribosomal proteins in this fraction was regarded as equimolar. Proteins of the small and large subunits had the same emPAI value. Moreover, in fraction 18, a high content of ribosome-associated proteins (EF-Tu and EF-Ts translation factors and Tig and DnaK chaperones) and HU protein was detected. It is known that HU protein, which is a bacterial histone-like protein, can bind both DNA and RNA [17]. It is possible that this protein can bind mRNA or rRNA within the ribosomes.

In fraction 18, we identified a GCW_03230 protein with a high emPAI value. This conservative protein of unknown function is common in many mycoplasmas. Considering the small size ( 74 amino acids), the feature of this protein is the extreme pI value (11.0), which makes the protein similar to ribosomal proteins. GCW_03230 is likely a new ribosomal protein. In frac- tion 18, a number of proteins with a relatively high emPAI value, which were not directly related to the process of translation (e.g., triosephosphate isomerase, thioredoxin, a number of proteins of unknown function), were also detected. On the one hand, their presence can be explained by nonspecific interactions with ribosomes after cell lysis. On the other hand, it has been shown recently that such proteins can modulate ribosome activity in vivo [11].

\section{Influence of secondary structures and ribosome binding site on mRNA abundance in the ribosome-bound pool}

Our results indicate that the mRNA abundance in the ribosome-bound fraction generally corresponds to the mRNA abundance in the total RNA fraction. However, some mRNAs were significantly more or less abundant in this fraction. Binding efficiency of mRNA to the ribosome was determined also by complementary interactions between the 3 ' end of $16 \mathrm{~S}$ rRNA and the ribosome binding site in the 5'-untranslated region (5'-UTR) of mRNA and by the presence of secondary structures in this region that mediate or block binding to ribosomes.

Using the RNA duplex program, we modeled in silico the interaction between the 3'-terminal region of $16 \mathrm{~S}$ rRNA (UUACCUCCUUUCU; underlined is the canonical ribosome binding site in E. coli) and the 25 -nucleotide region upstream of the start codon of each gene. Thus, we obtained results of the binding force of the 16S rRNA with the 5'-UTR of the corresponding mRNA. Spearman correlation between our evaluation of the capacity of the ribosome binding site and the abundance of the corresponding $\mathrm{mRNA}$ in the ribosome-bound RNA fraction was $0.39(p<0.01)$. We selected mRNA with more than 2-times up-abundance (19) and down-abundance (25) in the ribosome-bound RNA fraction compared to the total mRNA. The energy of duplex formation with the 3' end of $16 \mathrm{~S}$ rRNA in 5'-UTR of the up-abundant mRNAs was on average half that of down-abundant mRNAs (dG was -4.96 and $-2.52 \mathrm{kcal} / \mathrm{mol}$, respectively).

Despite the expected low efficiency of binding to ribosomes (dG>0), some mRNAs (e.g., GCW_02495 and putA) were more abundant in the ribosome-bound fraction than in the total RNA fraction. In the case of GCW_02495, this paradox can be explained by the fact that the GCW_02495 gene is expressed with polycistronic mRNA, together with the adjacent GCW_02490 gene that has a very effective ribosome binding site $(\mathrm{dG}=-11.8 \mathrm{kcal} / \mathrm{mol})$. Thus, the corresponding mRNA generally binds well with the ribosome.

Several mRNAs were less abundant in the ribosomebound fraction than in the total RNA fraction despite 
the predicted efficiency of ribosome binding. These mRNAs are GCW_00085, glpF, gyrA, gyrB, ruvA, potD, and $h r c A$. This behavior can be explained by the presence of certain secondary structures in the 5'-UTR of mRNA which prevent ribosome binding. Using the quickfold program, we calculated dG values of hairpin structure formation in the region of ribosome binding site and start codon using a sliding window of 30 nucleotides. As a result, we found that the $\mathrm{dG}$ value of the secondary structure near the start codon correlates with mRNA abundance in the ribosome-bound fraction (Fig. 2). The best correlation was identified in the range of $-21 \ldots+9$ nucleotides upstream of the start codon both for mRNA abundance in the ribosome-bound fraction and for the relative $\mathrm{mRNA}$ abundance in the ribosome-bound fraction regarding the total RNA level. Thus, the mRNA abundance in the ribosome-bound fraction in M. gallisepticum can be modulated by secondary structures in the start codon region.

\section{CONCLUSIONS}

Our results suggest that the amount of ribosome-bound mRNA in $M$. gallisepticum is largely determined by two parameters: (1) the level of gene transcription and (2) the efficacy of the complementary interaction between the 3'-end of $16 \mathrm{~S}$ rRNA and the ribosome binding site in the 5'-UTR of mRNA. We have developed a quantitative and reproducible method for obtaining the ribosome-bound fraction of mRNA from M. gallisepticum, which can be used for studying the process of translation in this bacterium.

The work was supported by the grant from the Russian Science Foundation (No 14-24-00159).
REFERENCES

1. Güell M. et al. Transcriptome complexity in a genome-reduced bacterium. // Science. 2009. V. 326. № 5957. P. 12681271.

2. Kühner S. et al. Proteome organization in a genome-reduced bacterium. // Science. 2009. V. 326. № 5957. P. 12351240 .

3. Karr J.R. et al. A whole-cell computational model predicts phenotype from genotype // Cell. 2012. V. 150. № 2. P. 389-401.

4. Fisunov G. et al. Core proteome of the minimal cell: comparative proteomics of three mollicute species // PLoS One. 2011. V. 6. № 7. P. e21964.

5. Moreno-Campuzano S., Janga S.C., Pérez-Rueda E. Identification and analysis of DNA-binding transcription factors in Bacillus subtilis and other Firmicutes-a genomic approach. // BMC Genomics. 2006. V. 7. P. 147.

6. Mazin P. V. et al. Transcriptome analysis reveals novel regulatory mechanisms in a genome-reduced bacterium // Nucleic Acids Res. 2014. V. 42. № 21. P. 13254-13268.

7. Faner M.A., Feigh A.L. Identifying and Characterizing HfqRNA Interactions // Methods. 2013. V. 63. № 2. P. 144-159.

8. Hillebrand A. et al. The seven E. coli ribosomal RNA operon upstream regulatory regions differ in structure and transcription factor binding efficiencies // Biol. Chem. 2005. V. 386. № 6. P. 523-534.

9. Moll I., Resch A., Bläsi U. Discrimination of 5'-terminal start codons by translation initiation factor 3 is mediated by ribosomal protein S1 // FEBS Lett. 1998. V. 436. № 2. P. 213-217.
10. Shi J. et al. SuhB is a novel ribosome associated protein that regulates expression of MexXY by modulating ribosome stalling in Pseudomonas aeruginosa. // Mol. Microbiol. 2015. V. 98. № 2. P. 370-383.

11. Fuchs G. et al. Proteomic Analysis of Ribosomes: Translational Control of mRNA populations by Glycogen Synthase GYS1 // J Mol Biol. 2011. V. 410. № 1. P. 118-130.

12. Lu P. et al. Absolute protein expression profiling estimates the relative contributions of transcriptional and translational regulation. // Nat. Biotechnol. 2007. V. 25. № 1. P. 117-124.

13. Nie L., Wu G., Zhang W. Correlation of mRNA expression and protein abundance affected by multiple sequence features related to translational efficiency in Desulfovibrio vulgaris: A quantitative analysis // Genetics. 2006. V. 174. № 4. P. 2229-2243.

14. Picard F. et al. Bacterial translational regulations: high diversity between all mRNAs and major role in gene expression. // BMC Genomics. 2012. V. 13. P. 528.

15. Ingolia N.T. Ribosome profiling: new views of translation, from single codons to genome scale. // Nat. Rev. Genet. Nature Publishing Group, 2014. V. 15. № 3. P. 205-213. 16. Gorbachev A.Y. et al. DNA repair in Mycoplasma gallisepticum // BMC Genomics. 2013. V. 14. P. 726.

17. Balandina A., Kamashev D., Rouviere-Yaniv J. The bacterial histone-like protein $\mathrm{HU}$ specifically recognizes similar structures in all nucleic acids. DNA, RNA, and their hybrids // J. Biol. Chem. 2002. V. 277 № 31. P. 27622-27628. 